\title{
耦合多水动力过程的二维山坡产汇流数值模型 及其在壤中流模拟中的应用
}

\author{
兰旻，胡宏昌*，田富强，胡和平 \\ 清华大学水利水电工程系水沙科学与水利水电工程国家重点实验室, 北京 100084 \\ *E-mail: huhongchang@ tsinghua.edu.cn
}

收稿日期: 2013-05-10; 接受日期: 2013-08-22

国家自然科学基金(批准号: 51190092, 51109110, 51222901)和水沙科学国家重点实验室统筹课题(批准号: 2012-KY-03)资助项目

\begin{abstract}
摘要 基于物理性水分运动方程的山坡产汇流数值模型是进行降雨产流机制和规律研究 的重要工具, 当前还缺乏耦合地表水、土壤水和地下水多水动力过程的、具有较高计算效率 和稳定性的二维山坡产汇流数值模型. 本研究耦合地表水流动的圣维南方程组、变饱和土壤 水分运动的 Richards 方程, 采用有限差分法, 对边界条件进行了改进, 建立了 THRM 模型, 对实验山坡实测壤中流过程有很好的模拟效果，通过数值试验揭示了边界条件、网格大小、 初始含水率等对计算稳定性和计算效果的影响规律, 所建模型对于山坡产流研究有应用价 值, 分析结论对壤中流数值模拟有参考意义.
\end{abstract}

关键词

山坡水文过程

壤中流

Richards 方程

渗流边界

数值稳定性

\section{1 引言}

山坡作为流域的基本构成单元，其水文响应特 性是流域水文学研究的中心问题之一. 逐步揭示山 坡水文过程的特性，进而探索流域不同尺度的水文 规律, 对水文机理的深入研究和流域水文学的发展 有重要意义. 目前对山坡水文过程的研究主要有理 论解析、室内及野外实验与观测、数值模拟等方式. 20 世纪六七十年代, 水文流域研究得到了广泛重视, 山 坡水文观测研究也随之长足发展, 在小流域降雨径 流观测的同时, 对山坡内部详细水文过程的观测也 进行了不少有益的尝试，但受制于技术手段对不同 的水流路径和滞留时间等的观测还十分有限 ${ }^{[1,2]}$. 室 内山坡水文人工试验则大多集中于土壤特征参数实 验, 对山坡水文过程的概化较为简单 ${ }^{[3]}$. 近年来, 随
着观测技术的进步和水文过程研究的深入, 许多学 者在野外实际流域开展了山坡尺度的精细观测与研 究, 如美国俄勒冈州的 H J Andrew 实验森林 ${ }^{[4]}$ 、佐治 亚州的 Panola 实验流域 ${ }^{[5]}$ 、阿拉斯加州南部的 Hecla Greens Creek 矿区实验山坡 ${ }^{[6]}$, 新西兰的 Maimai 实验 流域 ${ }^{[7]}$, 德国的 Weiherbach 实验流域 ${ }^{[8]}$ 等. 这些实验 区域依托已有的长系列水文和气象数据, 选取典型 山坡, 进行精细观测(包括地形、土壤、植被、基岩等 基础信息和地下水位、土壤含水率、山坡出流等实时 数据), 并结合同位素等示踪剂技术进行人工降雨或 分层注入等实验, 对降雨-径流过程中的路径、滞留时 间等进行观测与分析, 以求更深入了解山坡水文响应 的过程与特点. 随着研究的深入, 山坡整体地貌 ${ }^{[9 \sim 11]}$ 、 基岩微地形和基岩渗透性 ${ }^{[12]}$ 等传统山坡水文研究中 未考虑的因子被引入，进而提出了反映真实水文物

英文版发表信息： Lan M, Hu H C, Tian F Q, et al. A two-dimensional numerical model coupled with multiple hillslope hydrodynamic processes and its application to subsurface flow simulation. Sci China Tech Sci, 2013, 56: 2491-2500, doi: 10.1007/s11431-013-5347-6 
理过程且相对简化的山坡水文过程模型 ${ }^{[13]}$.

结合野外观测, 许多学者尝试利用数值模拟来 克服实验流域数量少、代表性差的局限. 虽然各种流 域性水文模型种类繁多, 但适用于山坡产流问题并 得到较多应用的模型很少. 杨志勇 ${ }^{[14]}$ 的 SAWH 模型, 通过概率密度函数描述了土壤饱和导水率和地形的 微观不均匀性, 并将山坡概化为沟道和坡地组成的 系统，通过沟道分布和坡地侧向坡度简化表征山坡 的地形特性. 但这种基于流域计算对山坡特性的模 拟都比较简单和概化, 无法清晰揭示山坡产流过程 的关键问题.

在针对山坡水文过程的数值模拟研究中, 基于 土壤水分运动 Richards 方程的经典土壤水分运动模 型 Hydrus ${ }^{[15]}$ 的应用较多, 其中代表性的是 Hopp 等 人 $^{[16]}$ 以实测数据为基准, 利用 Hydrus 模型模拟了 Panola 实验山坡的场次降雨径流过程, 并进行了 12 组虚拟实验, 分析了坡度、土壤厚度、降雨场次大小 和基岩渗透性 4 种山坡控制因素对山坡连通性和径 流系数等评价指标的影响, 揭示了坡度、土壤厚度与 降雨场次大小的耦合影响效应, 提出了地下饱和区 域的连通性可以用于描述不同类型的山坡响应. CATFLOW 模型则基于 2D Richards 方程, 也可以用 于山坡模拟, 被 Klaus 和 Zehe ${ }^{[17]}$ 用于对山坡快速流动 的研究. Harman 和 Sivapalan ${ }^{[18]}$ 在山坡尺度下, 以无 量纲手段, 引入特征时间, 基于 Boussinesq 方程和线 性水库概念构建了高度简化的概念性模型, 并在虚 拟实验中对导水率异质性的影响进行了探讨. 虽然 前人对山坡各种水流运动的耦合研究不少 ${ }^{[19]}$, 但目 前应用较广泛的模型如 Hydrus 等人只能进行壤中流 的计算，应用在部分无坡面产流的森林山坡地区.而 Harman 等人的模型则是基于特定水文过程分析的简 单模型, 难以推广应用于实际山坡.

壤中流模拟由于需要精细刻画山坡下垫面特性, 计算量大, 数值计算中涉及大型稀疏矩阵求解和高 度非线性等问题, 已有模型的计算效率较低, 在不同 网格划分和边界条件的组合下存在收玫性问题, 应 用受到很大限制. 已有文献中对于壤中流数值模拟 鲜有具体研究的叙述, 对影响模拟效果的因素如时 间和空间步长选取、边界条件等也少有探讨. 据此, 本文的研究目标是构建适用于山坡产汇流计算的多 水动力过程耦合模型, 在实际流域进行验证, 并针对 壤中流数值模拟的稳定性及其影响因素进行探讨,
为山坡产流理论研究提供分析工具.

\section{2 塊合多水动力过程的二维山坡产汇流数 值模型}

在已有土壤水分运动一维和二维数值模型

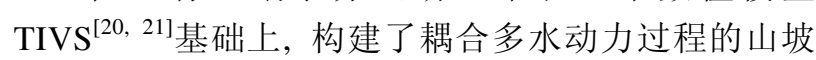
产流数值模型 Tsinghua Hillslope Runoff Model Based on Multiple Hydrodynamic Process(THRM), 模拟包括 山坡基岩的填洼-溢出(fill-and-spill) ${ }^{[22] 、}$ 基岩渗漏和 径流入渗(run-on)等山坡水文过程, 实现了坡面产流 与壤中流的耦合计算, 并通过变饱和土壤水分运动 方程实现非饱和土壤水流与饱和地下水流的耦合计 算. 同时, 实现了线性方程组的并行求解, 具有较好 的运算速度和计算精度.

\section{1 水分运动基本方程}

1) 入渗与 run-on 现象

根据 Horton 入渗理论, 当雨强超过入渗能力时, 超渗部分形成地面径流 ${ }^{[1]}$. 入渗能力 $f_{c}$ 一般可表示为 饱和导水率 $K_{s}$ 和土壤含水量 $\theta$ 的函数, 如 GreenAmpt 公式. 由于山区森林坡地土壤疏松, 饱和导水 率很高, 故本研究直接将入渗能力取为土壤饱和导 水率. 当降雨强度为 $r$ 时, 实际入渗率 $f=\min \left(K_{s}, r\right)$.

在饱和导水率较低的山坡中, 超渗部分将形成 坡面径流，同时会伴随有径流入渗(run-on)，即坡面 径流在下游局部土壤再入渗的现象 ${ }^{[23,24]}$. 本模型考 虑超渗条件下的 run-on 效应, 将其地表填洼部分处 理为下一步长的入渗 (以饱和导水率入渗), 剩余部分 成为坡面产流. 坡面产流与壤中流共同构成了山坡 产流, THRM 可以完成其耦合计算.

2) 坡面汇流

坡面汇流采用简化的 Saint Venant 方程, 采用运 动波方程进行计算 ${ }^{[25]}$, 摩擦坡度等于地面坡度

$$
\frac{\partial q}{\partial x}+\frac{\partial z_{h}}{\partial t}=i-f
$$

其中 $q=\beta z_{h}^{\gamma}, \gamma=\frac{1}{\delta} \sqrt{S_{0}}, q$ 为单宽流量, $x$ 为水平向 坐标(见图 1), $z_{h}$ 为山坡地表积水深度, $t$ 为时间, $i$ 为降 水量, $f$ 为入渗量, $\beta, \gamma$ 为参数, $\delta$ 为曼宁系数, $S_{0}$ 为摩擦 坡度。 


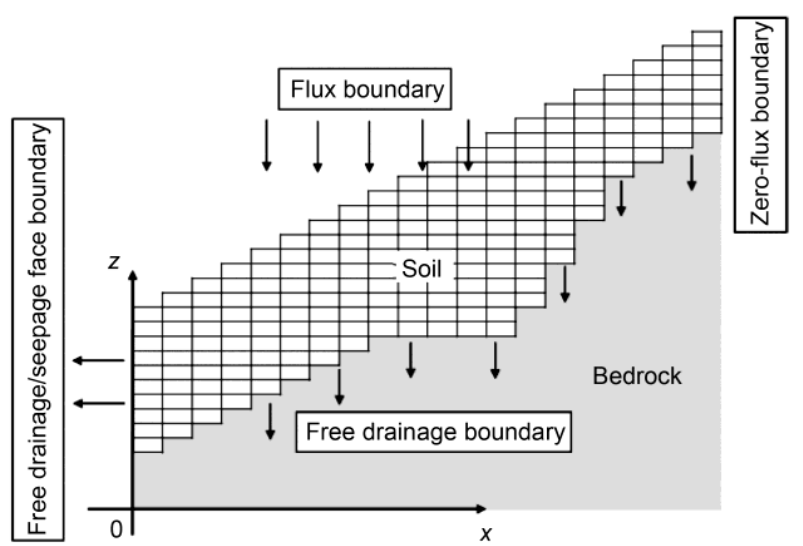

图 1 边界条件设置示意图

\section{3) 变饱和土壤水分运动}

土壤水分运动采用基于 $h$ 和混合式两种 Richards 方程计算，如下:

$$
\begin{gathered}
C(h) \frac{\partial h}{\partial t}-\frac{\partial}{\partial x}\left(K \frac{\partial h}{\partial x}\right)-\frac{\partial}{\partial z}\left(K \frac{\partial h}{\partial z}\right)-\frac{\partial K}{\partial z}-U=0, \\
\frac{\partial \theta}{\partial t}-\frac{\partial}{\partial x}\left(K \frac{\partial h}{\partial x}\right)-\frac{\partial}{\partial z}\left(K \frac{\partial h}{\partial z}\right)-\frac{\partial K}{\partial z}-U=0,
\end{gathered}
$$

其中 $h$ 为土壤水势, $\theta$ 为含水量, $x$ 和 $z$ 分别为水平向和 垂向距离(图 1), $t$ 为时间, $K$ 为土壤导水率, 是含水量 $\theta$ 的函数, $U$ 为源项, $C(h)$ 为比水容量 ${ }^{[26]}$.

\section{4) 边界条件}

在传统山坡模拟采用的边界条件组合基础上, 模型设定上边界为通量边界, 即入渗量 $f$; 下边界考 虑了基岩的渗透性, 设为自由出流边界; 坡顶为零通 量边界; 而坡脚则设置了自由出流与渗流两种边界 条件.

自由出流边界(Free Drainage Boundary)为定梯度 边界, 多用于土壤剖面较厚的情况, 常设置为单位梯 度, 即当网格土壤含水率 $\theta$ 大于残余含水率 $\theta_{r}$ 时产流,

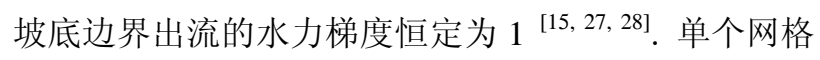
边界出流量 $q_{B}$ 计算式如下:

$$
q_{B}= \begin{cases}0, & \theta \leqslant \theta_{r}, \\ K(\theta) \times \frac{1}{\Delta x}, & \theta>\theta_{r},\end{cases}
$$

其中 $\Delta x$ 为计算空间离散后的网格水平向尺寸(详见 2.4 节).

渗流边界(Seepage Face Boundary)的设定为: 相 应边界土壤在达到饱和甚至特定积水条件下才出流. 即网格水头 $h$ 末达到阈值水头(用 $h_{s f}$ 表示, 非积水情
况下一般取 0$)$ 时出流为 0 , 达到后保持该阈值水头, 为定压水头边界, 单个网格边界出流量 $q_{B}$ 为

$$
q_{B}= \begin{cases}0, & h<h_{s f}, \\ K \times \frac{h}{\Delta x \times \xi} \times \frac{1}{\Delta x}, & h \geqslant h_{s f},\end{cases}
$$

其中 $\Delta x$ 为计算空间离散后的网格水平向尺寸.

由于达國值水头后的流量与网格宽度 $\Delta x$ 的二次 方成反比, $\Delta x$ 过大或过小可能导致瞬时流量异常而 无法稳定计算, 故在模型中引入边界梯度折减系数 $\xi$, $\xi>1$.

\section{2 土壤水力特征参数}

Richards 方程求解中用到的不饱和土壤的水力 特征参数在 THRM 中设置了 Broods-Corey 模型和 Van Genuchten 模型可供选用, 下文研究中采用 VG

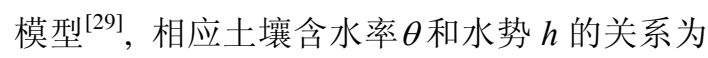

$$
\theta(h)= \begin{cases}\theta_{r}+\frac{\theta_{s}-\theta_{r}}{\left[1+|\alpha h|^{n}\right]^{m}}, & h<0, \\ \theta_{s}, & h \geqslant 0,\end{cases}
$$

其中 $\theta_{s}$ 为饱和含水率, $\theta_{r}$ 为残余含水率, $n, m, \alpha$ 为经验 系数.

土壤不饱和导水率 $K(h)$ 和比水容量(Soil Water Capacity) $C(h)$ 的计算公式如下:

$$
\begin{gathered}
K(h)=K_{s} S_{e}^{l}\left[1-\left(1-S_{e}^{1 / m}\right)^{m}\right]^{2}, \\
C(h)=\frac{-\alpha m\left(\theta_{s}-\theta_{r}\right)}{1-m} S_{e}^{1 / m}\left(1-\left(S_{e}\right)^{1 / m}\right)^{m},
\end{gathered}
$$

其中 $S_{e}$ 为有效饱和度, $S_{e}=\frac{\theta-\theta_{r}}{\theta_{s}-\theta_{r}}=\left(1+|\alpha h|^{n}\right)^{-m}, l$ 为经验系数.

\subsection{Richards 方程求解选择算法}

基于 $h$ 和混合式 Richards 方程求解的区别是迭代 过程中 $C(h)$ 高度非线性的特征 ${ }^{[30]}$ 是否保留. 如果不 考虑迭代, 则基于 $h$ 和混合式 Richards 两种方程的求 解方法是完全一致的 ${ }^{[30,31]}$. 当采取迭代算法时, 基于 $h$ 的 Richards 方程求解在一个时间步长内不考虑 $C(h)$ 的非线性特征, 因此会产生较大的误差, 但其运行步 长数和时间少于混合式 Richards 方程的求解. 结合两 种方程的特点, 可采用基于 $h$ 变化的选择算法或基于 饱和状态的选择算法两种不同处理方式. 本研究综 
合两种选择算法, 二者满足其一即由采用混合式改 用基于 $h$ 的 Richards 方程计算, 有效提高了计算效率 和稳定性.

\section{1）基于 $h$ 变化的选择算法}

在一个迭代步长内, 若 $h$ 的变化足够小, $C(h)$ 在 步长内的变化也足够小, 非线性化程度较低, 基于 $h$ 的 Richards 方程可以得到较好的水量平衡效果; Hao 等人 ${ }^{[32]}$ 研究表明，相同的误差阈值下，相对于混合型 Richards 算法而言, 选择算法对大多数土壤都有较好 的收玫性和水量平衡精度, 并且对土壤属性为分层 结构的模拟效果更好, 在研究中提出 $3 \mathrm{~cm}$ 作为土壤 水势的变化阈值.土壤水势变化阈值的选择跟土壤 类型，计算网格大小，误差控制和时间步长控制等都 有重要的关系. 基于 $h$ 变化的选择算法为特定条件下 Richards 方程的求解提供了选择, 但数值实验显示其 水量平衡误差随水势阈值的增大而增大, 在应用中 应注意.

\section{2) 基于饱和状态的选择算法}

完全饱和状态下, $C(h)=0$, 求解不受算法的影响; 在近似饱和状态, $C(h)$ 变化也很小, 非线形化程度较 低, 使用基于 $h$ 的 Richards 方程求解可以取得较好的 水量平衡效果; 如果土壤水势小于控制阈值 $h_{0}$, 则使 用混合式 Richards 方程求解，反之则采用基于 $h$ 的 Richards 方程求解. Sadegh ${ }^{[33,34]}$ 在研究中应用了该方 法, 控制阈值 $h_{0}=-2.5 \mathrm{~cm}$, 取得了较好的模拟效果. 随着 $h_{0}$ 的减小, 水量平衡误差逐渐增大.

\section{4 时空离散与数值解法}

模拟区域进行矩形剖分，在不同地形的模拟中 可以更好地平衡精度与计算效率. 考虑下垫面条件 的异质性, 各网格点相应土壤参数值均可以独立设 定. 方程离散采用网格中心差分格式, 可将土壤水分 运动方程(以基于 $h$ 的 Richards 方程为例)在时间和空 间上离散如下:

$$
\begin{aligned}
& C\left(h_{i, j}^{a+1, b+1}\right)\left(\frac{h_{i+1, j}^{a+1, b+1}-h_{i+1, j}^{a}}{\Delta t}\right)-\frac{1}{\Delta z} \bar{K}_{i+\frac{1}{2}, j}^{a+1, b}\left(\frac{h_{i+1, j}^{a+1, b+1}-h_{i, j}^{a+1, b+1}}{\Delta z_{i}}+1\right) \\
& +\frac{1}{\Delta z} \bar{K}_{i-\frac{1}{2}, j}^{a+1, b}\left(\frac{h_{i, j}^{a+1, b+1}-h_{i-1, j}^{a+1, b+1}}{\Delta z_{i-1}}+1\right)-\frac{1}{\Delta x} \bar{K}_{i, j+\frac{1}{2}}^{a+1, b} \frac{h_{i, j+1}^{a+1, b+1}-h_{i, j}^{a+1, b+1}}{\Delta x_{i}} \\
& +\frac{1}{\Delta x} \bar{K}_{i, j-\frac{1}{2}}^{a+1, b} \frac{h_{i, j}^{a+1, b+1}-h_{i, j-1}^{a+1, b+1}}{\Delta x_{i-1}}=0 .
\end{aligned}
$$

类似地，可将汇流运动波方程离散如下：

$$
\begin{aligned}
& z_{h j}^{a}+\alpha \frac{\Delta t}{\Delta x}\left(z_{h j}^{a}\right)^{\gamma}-z_{h j}^{a-1}-\alpha \frac{\Delta t}{\Delta x}\left(z_{h j-1}^{a}\right)^{\gamma} \\
& -(i-f)_{j}^{a}=0,
\end{aligned}
$$

其中各上下标中 $i, j$ 为相应网格的垂向、水平向坐标, $a$ 为当前的时间标示, $b$ 为当前的迭代步数.

模型的数值离散格式采用有限差分方法. 由于 水分运动方程对应于计算时间的状态变量是隐式和 非线性的，利用隐式差分求解，需要利用迭代算法. 模型采用具有较好迭代效率的 Newton-Raphson 迭代 技术, 迭代计算采用变步长方法, 每个输入步长内初 始的计算步长可设定. 如果计算步长内的变量(水势 或水分)变化值超过设定的阈值，则模型调整计算步 长，将其缩短到现有时间步长的 1/1.3. 计算步长内 求解线性方程组得到变量的新值, 确认其变化值, 如 果变化值超过设定的计算误差, 则利用计算的新值 更新土壤水力传导度、土壤比水容量和该迭代步长的 土壤含水率, 得到新的线性方程组, 继续迭代求解; 如果变量的变化值小于设定的计算误差, 则停止迭代.

二维水分运动方程有 5 个状态变量(即 $h_{i-1, j}, h_{1, j}$, $\left.h_{i+1, j}, h_{i, j-1}, h_{i, j+1}\right)$, 利用多变量 Newton-Raphson 方法进 行求解, 所得线性方程组可以写成五角矩阵的形式. 研究选用 MUMPS(A MUltifrontal Massively Parallel Sparse Direct Solver)软件包 ${ }^{[35]}$ 求解线性方程组. 该软 件包基于 Fortran90 编写, 并且有 $\mathrm{C}$ 语言的接口, 可 以实现稀疏线性方程组的序贯和并行求解.

\section{3 实验流域与数据}

选取美国 Panola 流域(Panola Mountain Research Watershed, PMRW) 的实验山坡 ${ }^{[16,36]}$ (以下简称 Panola 山坡)作为研究区域, 以验证和分析模型对山坡水文 响应的模拟.

PMRW 位于美国佐治亚州佐治亚山麓，属于亚 热带湿润气候, 年平均气温 $16.3^{\circ} \mathrm{C}$, 年平均降雨量 $1240 \mathrm{~mm}$, 降雨量在年内分布比较均匀. Panola 山坡 位于 PMRW 西侧, 如图 2 圆圈所示，长约 $48 \mathrm{~m}$, 宽约 $28 \mathrm{~m}$, 平均坡度 $13^{\circ}$, 坡顶为部分出露岩层, 坡底中 段人工挖掘了深至基岩的 $20 \mathrm{~m}$ 出流量测量沟槽.

山坡地表地形较为平坦，但基岩地形很不规则， 基岩岩性以微风化的花岗岩为主.土壤类型为砂壤 土, 饱和导水率较大, 表层有高达 $3 \mathrm{~m} / \mathrm{h}$ 的瞬时实测 
值, 平均饱和导水率约为 $0.64 \mathrm{~m} / \mathrm{h}$. 壤中流占径流主 导, 基本无坡面流产生. 选取大孔隙流较少的沟槽 $11 \mathrm{~m}$ 处的剖面进行计算, 如图 3 所示, 相应地表和基 岩地形剖面线如图 4 所示, 数据为 $1 \mathrm{~m} \times 1 \mathrm{~m}$ 的 DEM, 由 $2 \mathrm{~m} \times 2 \mathrm{~m}$ 的实地量测结果插值所得. 土壤厚度范围 为 $0.3 \sim 1.0 \mathrm{~m}$, 沿坡长方向每隔 2 4 $\mathrm{m}$ 分别有 $5,15,30$, $50 \mathrm{~cm}$ 深度的土壤含水率实测值.

\section{4 结果与讨论}

\section{1 场次过程模拟}

为了验证模型的有效性, 选取 Panola 地区中等

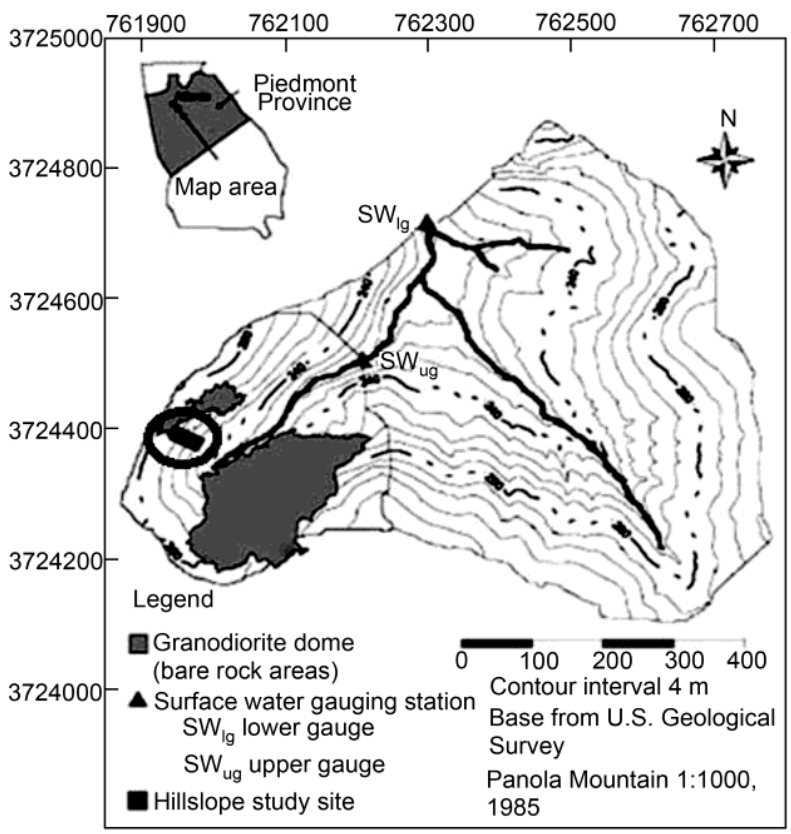

图 2 Panola 研究流域(PMRW)示意图

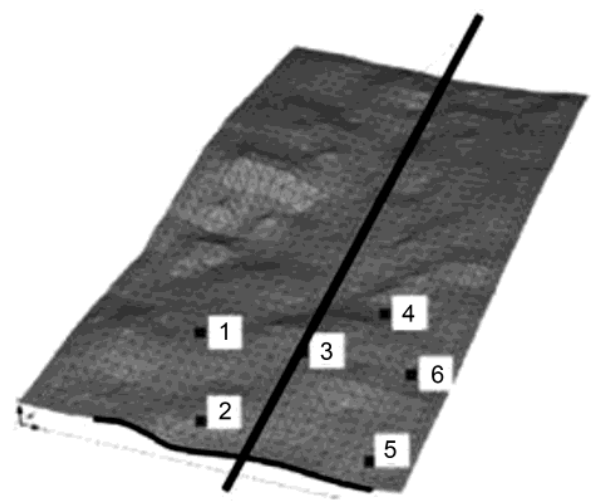

图 3 Panola 山坡示意 ${ }^{[16]}$

黑线所示位置为计算剖面选取处

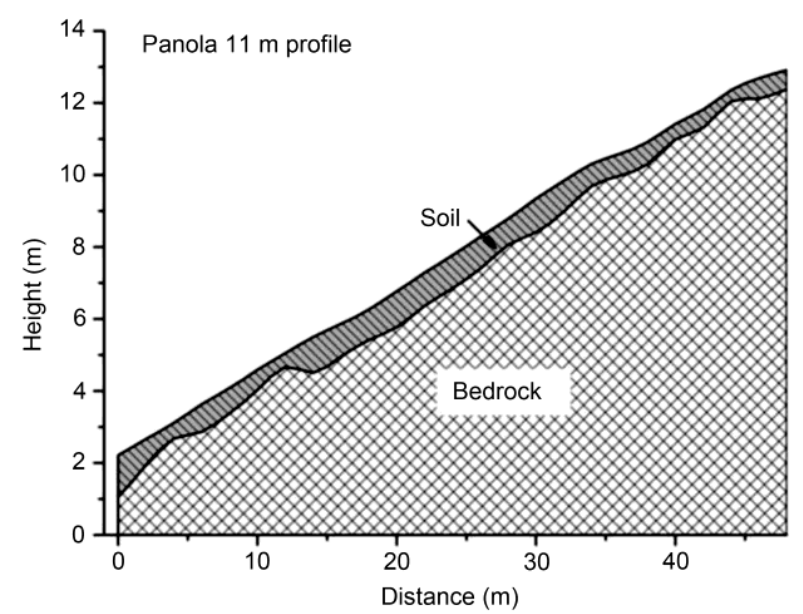

图 4 计算剖面(Panola 山坡 $11 \mathrm{~m}$ 处)地形示意图 阴影部分为土壤

降雨量的典型降雨过程, 2002 年 2 月 6 7 日的场次降 雨, 该场次总降雨量为 $58.9 \mathrm{~mm}$, 模拟时段为 2002 年 2 月 6 19 日, 不考虑蒸散发. 根据实测地形划分网格, 利用 Kriging 法由实测点数据获得全剖面各网格的土 壤含水量值. 模拟采用的其他参数, 如基岩渗透性、 土壤导水率等相关参数, 均在实测数值基础上率定, 如表 1 所示. 计算采用 10 核 Intel Xeon E7-4850 中央 处理器、 $64 \mathrm{G}$ 内存服务器, Ubuntu 系统. 结果表明, 各次模拟的水量平衡结果精度很高, 但自由出流和 渗流两种边界条件下流量过程线的表现有明显区别, 如图 5 所示.

自由出流边界条件下, 模拟结果相应的纳什效 率系数为 0.66. 相比实测值, 流量过程总体趋势一致, 但峰值后下降迅速, 且在计算时段结束时退水过程 仍在持续. 分析可知, 非干旱地区土壤初始含水率很 难低于残余含水率，因此基于自由出流方法计算的 边界出流将持续存在，不符合实际情况.

渗流边界条件下, 不考虑边界梯度折减(即(5)式 中的 $\xi=1$ )的模型纳什效率系数 NSE 为 0.90 , 计算时 长为 $1342.26 \mathrm{~s}$; 考虑了边界梯度折减 $(\xi=13)$ 的纳什效 率系数为 0.94 , 计算时长为 $125.82 \mathrm{~s}$. 率定得到的参 数如基岩渗透性(表示为 $L$, 即基岩导水率与土壤导 水率的比值)、土壤相关参数等大部分都与实测值十 分接近, 如表 1 所示. 与实测接近的计算参数和较好 的模拟结果显示, 在考虑了梯度折减的渗流边界条 件下，模型能够对山坡得到理想的单场次模拟效果 和计算效率. 因此, 此后渗流边界计算均采用考虑 $\xi$ 


\section{表 1 模型主要参数}

\begin{tabular}{|c|c|c|c|c|c|c|c|c|}
\hline Item & $K_{\mathrm{s}}(\mathrm{cm} / \mathrm{s})$ & $\overline{\theta_{\mathrm{s}}}$ & $\overline{\theta_{\mathrm{r}}}$ & $L$ & $\alpha\left(\mathrm{cm}^{-1}\right)$ & $m$ & $n$ & NSE \\
\hline Observed value & $0.02-0.08$ & $0.47-0.51$ & - & $\sim 0.001$ & - & - & - & - \\
\hline Free drainage boundary & 0.06 & 0.46 & 0.21 & 0.002 & 0.02 & 0.5 & 2 & 0.66 \\
\hline Seepage face boundary with $\xi=1$ & 0.04 & 0.46 & 0.21 & 0.002 & 0.02 & 0.5 & 2 & 0.90 \\
\hline Seepage face boundary with $\xi=13$ & 0.06 & 0.46 & 0.21 & 0.002 & 0.02 & 0.5 & 2 & 0.94 \\
\hline
\end{tabular}

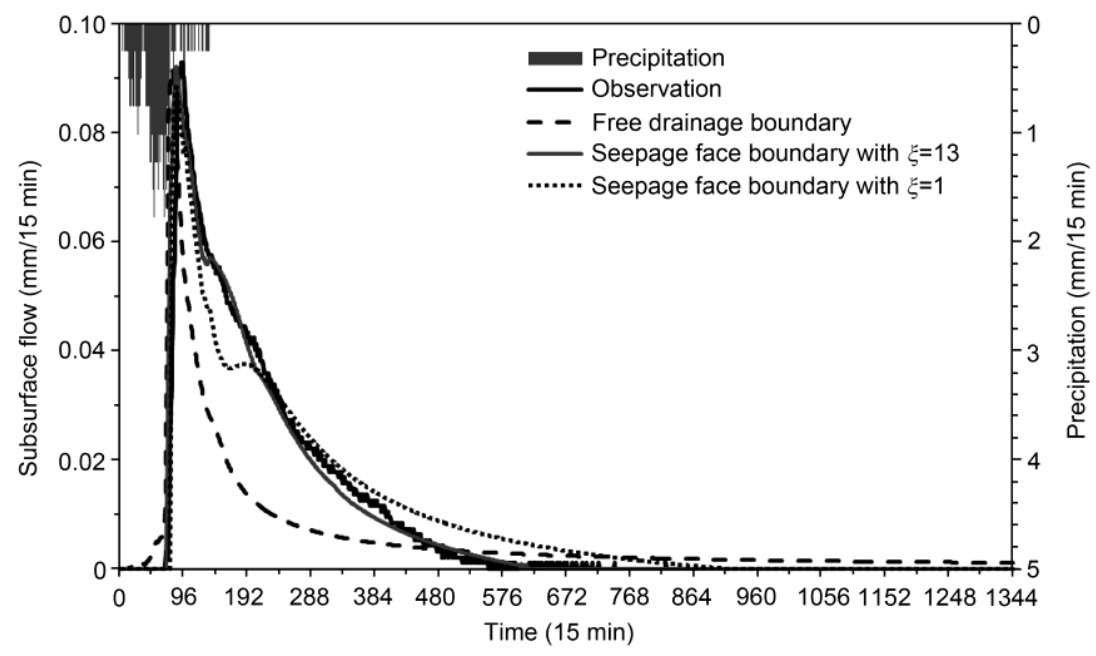

图 53 种边界降雨径流模拟结果

优化后的边界条件.

对渗流边界计算结果进行分析，可得相应剖面 水量平衡模拟结果如表 2 所示.

由表 2 结果可知, 在 Panola 山坡, 由于山坡土层 薄、地形坡度明显, 加之森林地区土壤导水率较大, 即使基岩导水率只有土壤导水率的千分之二, 基岩 渗漏量仍高达坡脚出口处径流量的 4 倍. 相似结果在 国外另外几个实验山坡如新西兰的 MaiMai 实验流域 ${ }^{[12]}$, 美国的 H.J Andrew 实验流域 ${ }^{[13]}$, 日本的 Kiryu 实 验流域 ${ }^{[37]}$ 等也都有发现, 体现出即使不是喀斯特地 区，基岩渗漏在森林地区也仍是山坡水量平衡的重 要组成.

\section{2 边界条件的影响}

自由出流边界虽然适用于土壤水分运动模拟, 但由上述研究可知, 应用于山坡水分运动模拟具有

\section{表 2 山坡水量平衡对比(单位: $\mathbf{m m}$ )}

\begin{tabular}{ccc}
\hline Item & Observation & Simulation \\
\hline Precipitation & 58.928 & - \\
Changes of soil water storage & -6.383 & -5.192 \\
Runoff depth & 12.521 & 12.448 \\
Bedrock leakage & 52.790 & 51.673 \\
\hline
\end{tabular}

局限性, 将导致结果出现峰值下降过快、退水段无限 延长等情况，与实际山坡响应特点不符，山坡水分运 动模拟应使用渗流边界条件. 因此, 本文将进一步探 讨渗流边界模拟中为数值计算稳定高效而引入的边 界梯度折减系数 $\xi$.

在 4.1 节的模拟结果中可知, 采用不考虑边界梯 度折减的模拟比考虑折减时的计算时间大了一个数 量级, 且饱和导水率等参数值只有在较小范围内时 才能稳定计算. 这是由于坡度的存在和水分运动变 化的增大, 需要尽量缩小时间步长和最小迭代步长 才可能稳定计算, 导致计算效率低下, 计算结果稳定 性和适用性较差. 考虑边界梯度折减系数 $\xi$ 的渗流边 界, 相当于对边界出流进行缓冲, 保证了计算的稳定 和精度, 提高了计算效率. 如图 6 和表 3 所示, 仅改 变梯度折减系数, 对水量平衡尤其是土壤含水率变 化的影响可忽略, 流量过程基本一致, 但取值差别较 大时对峰值有一定影响.

为保证计算的稳定性和峰值的合理性, 进一步 研究分析了不同网格大小稳定计算所需的边界梯度 折减系数 $\xi$, 结果表明, 当网格边长为 $20 \mathrm{~cm}$ 时, 保证 计算稳定的 $\xi$ 可行取值区间为 $[8,13]$, 而网格边长为 
表 3 不同边界梯度折减系数条件下的水量平衡结果 (单 位: $\mathbf{m m}$ )

\begin{tabular}{ccccc}
\hline \multirow{2}{*}{ Item } & \multirow{2}{*}{ Observation } & \multicolumn{3}{c}{ Simulation } \\
\cline { 3 - 5 } & & $\xi=10$ & $\xi=18$ & $\xi=28$ \\
\hline Changes of soil water & -6.383 & -5.194 & -5.194 & -5.194 \\
storage & & & & \\
Runoff depth & 12.521 & 12.813 & 12.510 & 12.167 \\
Bedrock leakage & 52.790 & 51.308 & 51.610 & 51.952 \\
\hline
\end{tabular}

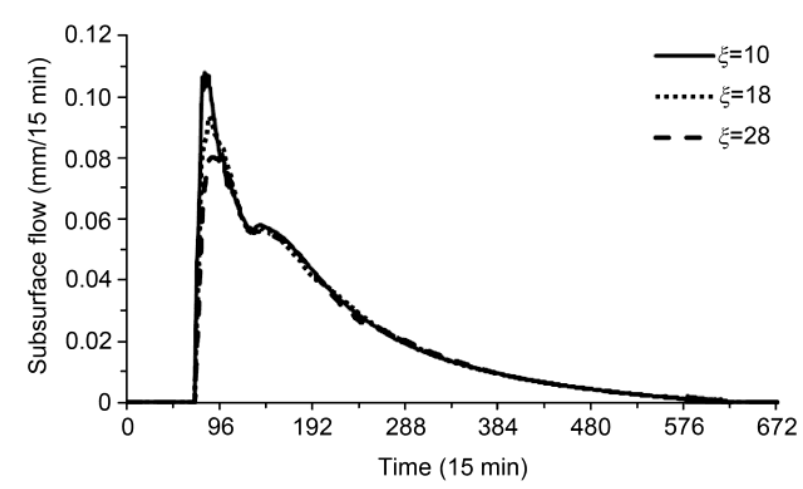

图 6 不同边界梯度折减系数模拟结果比较

$10 \mathrm{~cm}$ 时其可行区间为 $[10,28]$, 网格边长为 $5 \mathrm{~cm}$ 时, 其可行区间为 $[13,58]$. 网格越小, 边界梯度折减系 数的适用范围越大, 且下边界越大.

\section{3 网格大小的影响}

实际山坡模拟中，网格大小既影响对实际地形 和下垫面异质性的刻画, 又与模型效率直接相关. 上 文对出流折减系数的探讨也体现了数值计算中计算 网格尺寸变化带来的模拟表现的差异. 本文选用 5 , $10,20 \mathrm{~cm}$ 三种网格剖分尺寸, 进一步对网格尺寸的 影响进行分析讨论.

1) 计算时长

模拟长度为 $48 \mathrm{~m}$ 的 Panola 山坡剖面, $20 \mathrm{~cm}$ 网格 计算总时长约为 $29.71 \mathrm{~s}, 10 \mathrm{~cm}$ 网格计算总时长约为 $204.89 \mathrm{~s}, 5 \mathrm{~cm}$ 网格计算总时长约为 $1300.70 \mathrm{~s}$, 随着 网格的细化, 计算时长成数量级地增长. 由此可见, 权衡选取适当大小的计算网格对高效模拟实际流域 意义重大.

2) 水量平衡与水量过程线特性

水量平衡与流量过程是判断水文模拟效果的重 要依据. 按网格尺寸由大到小, 出流折减系数 $\xi$ 取为 13 时, 三者的水量平衡和流量过程如表 4 和图 7 所 示.

由此可知, 随着网格细化, 土壤含水量增加, 径
流量增加, 基岩渗漏量减少, 洪峰形状和退水曲线趋 势基本一致, 但 3 种尺寸模拟结果间仍有明显差异. 究其原因, 3 种尺寸网格模拟时均根据实测地形划分 网格，由于山坡地表和基岩地形的不规则，不同网格 大小影响了土壤的计算面积大小和基岩地形的表达, 如图 8 所示, $a$ 为 3 种网格大小地形剖分的不同结果 示意, $b$ 为在 $20 \mathrm{~cm}$ 网格基础上细分小网格示意. 最大 最小网格间土壤剖面面积相差 $1825 \mathrm{~cm}^{2}$. 同时, 初始 含水率数据为直接根据实测值和网格尺寸进行 Kriging 展开所得, 不同网格大小情况下 Kriging 插值 所得的平均土壤含水率和分布也存在差异.

为消除剖面面积的差异, 以 $20 \mathrm{~cm}$ 网格所得山坡 土壤剖面为基准，再将此剖面分别细分为 $10,5 \mathrm{~cm}$ 的 网格, 使三者土壤剖面面积相等, 可得计算结果如图 9 所示, 峰值差距有所缩小, 但并不明显. 可见在不 规则地形状态下, 不同网格大小造成的剖面面积差 异对水量平衡有一定影响, 而对流量过程的模拟影 响不大.

在面积相等的基础上将三种网格的初始含水率 均取同一系列值(将经过 Kriging 插值的 $20 \mathrm{~cm}$ 网格的 初始土壤含水率分别复制拆分为适用于 10 和 $5 \mathrm{~cm}$ 网 格计算的数据组), 如图 10 所示, 三者水量过程线形 状及峰值已经非常接近. 表明数值计算中网格尺寸 对出流结果的影响主要来自初始含水率数据处理误 差, 而模型对不同网格尺寸的模拟非常稳定.

\section{表 4 不同网格大小水量平衡(单位: $\mathrm{mm}$ )}

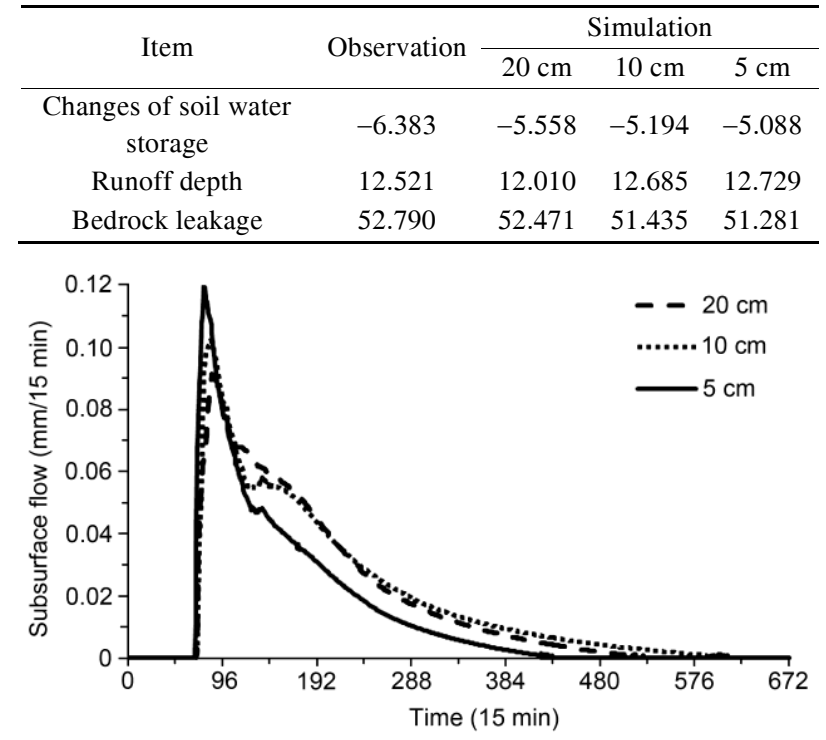

图 7 不同网格大小条件下的流量过程线比较 


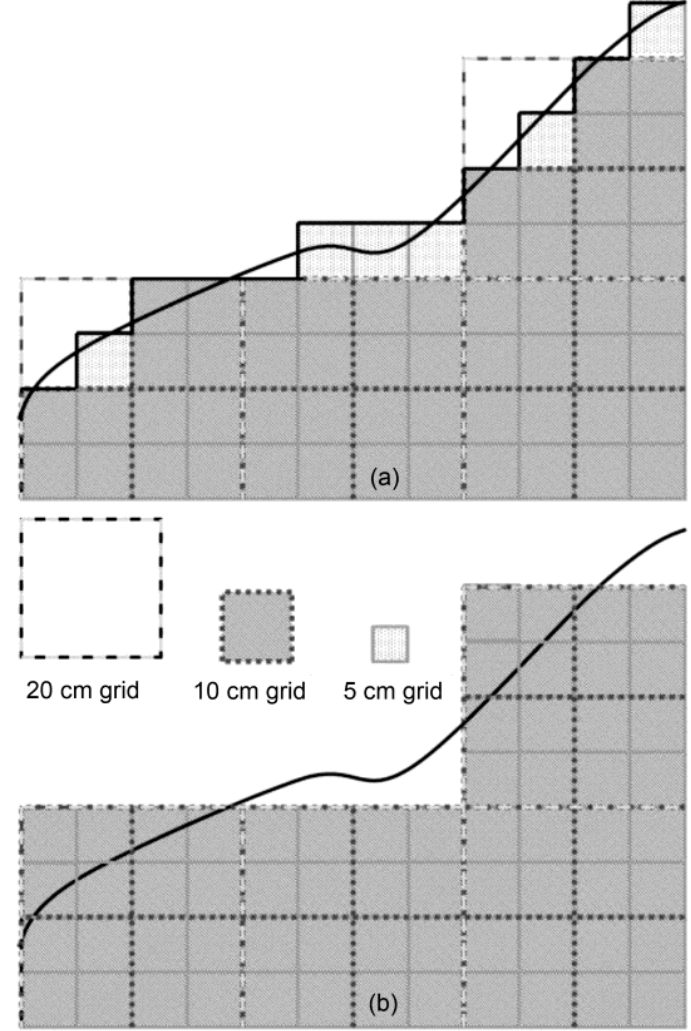

图 8 不同网格大小对不规则地形的表达

(a) 3 种网格大小地形剖分的不同结果示意; (b) $20 \mathrm{~cm}$ 网格基础上 细分小网格示意

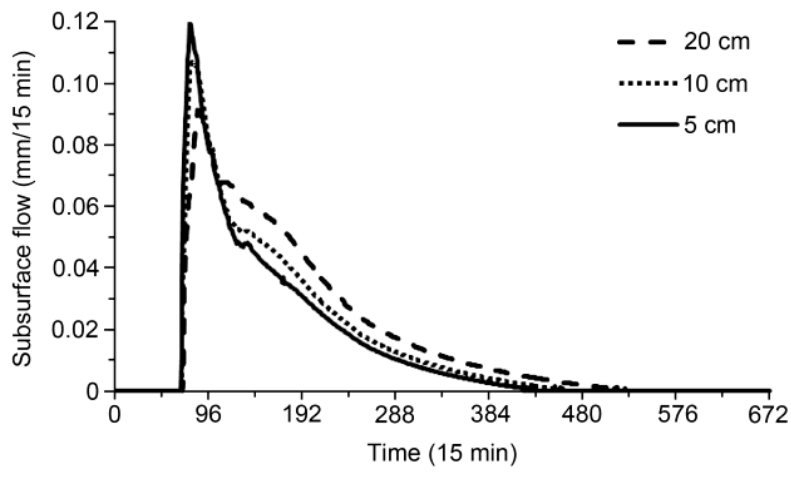

图 9 相等面积下不同网格大小流量过程线

\section{4 初始含水率的影响}

1) 初始含水率克里金展开的误差影响

克里金插值方法(Kriging)利用实测的有限个已 知值，通过考虑各测点之间的空间位置、相关程度等 的不同，对每一个观测资料赋予一定的权重系数，进 行滑动加权平均, 得到其他位置的估计值, 是土壤水 分研究中常用的插值方法 ${ }^{[8]}$. 然而, 在利用 Kriging

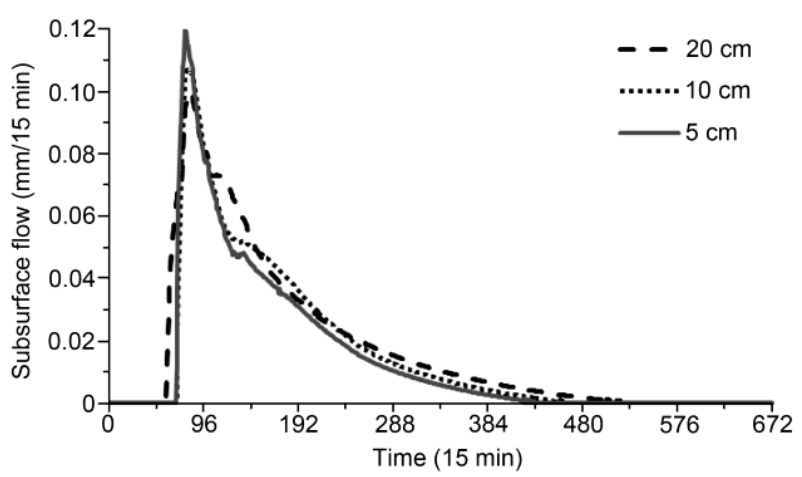

图 10 相等面积、相同初始含水率下不同网格大小流量过 程线

插值方法计算整个剖面各网格点土壤初始含水率时, 不同的网格尺寸使 Kriging 展开得到不同的初始土壤 含水率分布和平均值, 4.3 中的结果显示它对模型的 模拟结果产生了显著影响.

2) 初始含水率分布的影响

在考虑排除初始含水率误差的作用，只探讨网 格大小影响时，有两种不同的处理方式：一种是将整 个土壤剖面各网格的初始含水率统一设定为均一值 (图 11 中简称为均一); 另一种是考虑土壤含水率分 布, 采用将实测值 Kriging 插值后的同一组土壤含水 率数据(图 11 中简称为非均).

在渗流边界条件下，对以上两种不同初始土壤 含水率处理方法进行分析, 可得不同网格大小相应 流量过程和累加结果如下.

渗流边界下初始土壤含水率的分布对水量平衡 和流量过程线的总体趋势并没有明显的影响，总体 上与自由出流边界的特性一致. 这与 Tromp 和 Mcdonnell ${ }^{[38]}$ 对土壤含水率的空间相关性研究和 Hopp 和 Mcdonnell ${ }^{[16]}$ 的虚拟山坡数值实验所得结论 相符. 但本研究中, 基岩地形不均匀, 流量过程线在 退水中段产生了细微差别, 尤其是 $20 \mathrm{~cm}$ 网格下, 大 网格放大了过程中的变化, 非均一初始含水率时在 退水段出现了较为明显的峰值, 而均一初始土壤含 水率所得流量过程线在三种网格下全过程都十分平 滑, 实际流量过程则介于这二者之间. 因此, 在模拟 区域尺度较小、需要考虑流量过程细微变化时, 非均 一的土壤与基岩起伏共同造成的微小变化是无法直 接用均一土壤替代计算的.

研究显示了初始土壤含水率(前期降水量)对模 型模拟的重要影响. 初始土壤含水率的平均值影响 

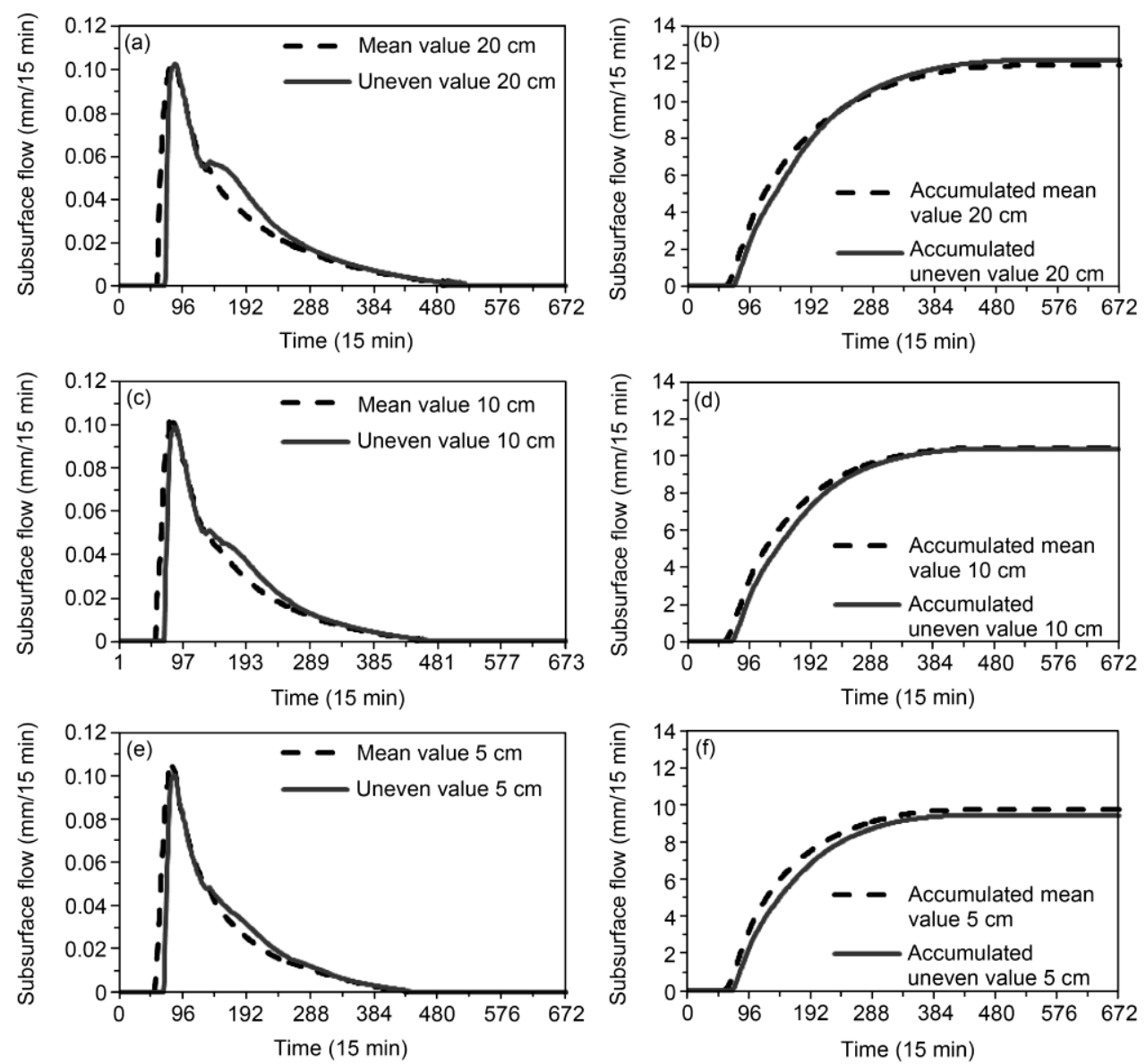

图 11 渗流边界均匀与非均匀初始含水量计算结果

模型的水量平衡和流量过程线的形状，与山坡出流 量成正相关; 而平均值相同时，其分布模式总体上与 水量平衡和出流过程关系不大, 但在某些特定过程 有细微差别, 可能是特定基岩地形与该区域土壤含 水率共同作用的结果, 有待进一步研究.

\section{5 结论}

本文构建了耦合多水动力过程的二维山坡产流 数值模型 Tsinghua Hillslope Runoff Model Based on Multiple Hydrodynamic Process(THRM), 可以实现对 山坡水文响应的精细模拟. 采用实际流域数据的验 证显示, 模型在实测参数的基础上微调即能对场次 过程实现较高精度的重现, 并较好地模拟了基岩渗 漏在山坡尺度水量平衡中的重要作用. 通过对土层 较薄的实际山坡模拟, 研究发现采用渗流边界比自 由出流边界对山坡水文响应过程的模拟更加准确,
而对渗流边界考虑梯度折减系数后模型计算更加稳 定高效, 计算时长缩短了一个数量级. 而山坡数值模 拟在考虑基岩渗漏和不规则坡面、基岩地形时，需要 注意网格大小与梯度折减系数之间的协调. 剖分时 采用的网格尺寸若成倍增大, 则计算时长成数量级 减小. 网格越大, 土壤含水量减少越明显, 径流量减 少, 基岩渗漏量增加, 洪峰形状和退水曲线趋势基本 一致, 但流量过程线仍有明显差异. 分析显示, 不同 网格尺寸的不同模拟结果, 主要是由于不同网格大 小分别利用 Kriging 方法获得土壤初始含水量时产生 的误差所致, 而不同网格大小造成不规则土壤剖面 的计算面积差异也对峰值有一定影响, 模型本身的 数值计算对网格尺寸并不敏感. 模型的模拟结果显 示了初始土壤含水率对山坡水文响应模拟的重要作 用, 但进一步分析显示, 初始土壤含水率的作用主要 源自平均值的变化，而相同均值下其分布对水量平 衡和流量过程线的总体趋势并没有明显的影响. 
致谢＼cjkstart感谢 Jeffery McDonnell 教授和 Julian Klaus 博士为本研究提供基础数据和良好建议.

\section{参考文献}

1 Kirkby M J. Hillslope Hydrology. Chichester: John Wiley \& Sons Ltd, 1978. 389

2 顾慰祖, 陆家驹, 唐海行, 等, 水文实验求是传统水文概念——纪念中国水文流域研究 50 年、滁州水文实验 20 年. 水科学进展, 2003 , 14: $368-378$

3 Hewlett J D, Hibbert A R. Moisture and energy conditions within a sloping soil mass during drainage. J Geophys Res, 1963, 68: 1081-1087

4 Mcguire K J, Weiler M, Mcdonnell J J. Integrating tracer experiments with modeling to assess runoff processes and water transit times. Adv Water Res, 2007, 30: 824-837

5 Freer J, Mcdonnell J J, Beven K J, et al. The role of bedrock topography on subsurface storm flow. Water Resour Res, 2002, $38: 1269$

6 Hopp L, Harman C, Desilets S L E, et al. Hillslope hydrology under glass: Confronting fundamental questions of soil-water-biota co-evolution at biosphere 2. Hydrol Earth Syst Sci, 2009, 13: 2105-2118

7 Weiler M, Mcdonnell J J. Conceptualizing lateral preferential flow and flow networks and simulating the effects on gauged and ungauged hillslopes. Water Resour Res, 2007, 43: W3403

8 Bárdossy A, Lehmann W. Spatial distribution of soil moisture in a small catchment. Part 1: Geostatistical analysis. J Hydrol, 1998, 206: $1-15$

9 Weiler M, Mcdonnell J. Exploring the first order controls of hillslope scale $\mathrm{n}$ and doc flushing: A virtual experiment approach. Presentation at the AGV Fall Meeting. San Francisco, 2002

10 Weiler M, Mcdonnell J J. Testing nutrient flushing hypotheses at the hillslope scale: A virtual experiment approach. J Hydrol, 2006, 319 : 339-356

11 Troch P A. Hillslope-storage boussinesq model for subsurface flow and variable source areas along complex hillslopes: 1. Formulation and characteristic response. Water Resour Res, 2003, 39

12 Graham C B, Woods R A, Mcdonnell J J. Hillslope threshold response to rainfall: (1) A field based forensic approach. J Hydrol, 2010, 393: 65-76

13 Graham C B, Mcdonnell J J. Hillslope threshold response to rainfall: (2) Development and use of a macroscale model. J Hydrol, 2010, 393 $77-93$

14 杨志勇. 基于概率描述的宏观尺度空间均化流域水文模型研究. 北京: 清华大学, 2007

15 Šimůnek J, Šejna M. Hydrus technical manual. 2ed eds. 2011

16 Hopp L, Mcdonnell J J. Connectivity at the hillslope scale: Identifying interactions between storm size, bedrock permeability, slope angle and soil depth. J Hydrol, 2009, 376: 378-391

17 Klaus J, Zehe E. Modelling rapid flow response of a tile-drained field site using a 2d physically based model: assessment of 'equifinal' model setups. Hydrol Process, 2010, 24: 1595-1609

18 Harman C, Sivapalan M. A similarity framework to assess controls on shallow subsurface flow dynamics in hillslopes. Water Resour Res, 2009, 45: W01417

19 Freeze R A. Role of subsurface flow in generating surface runoff: 2 Upstream source areas. Water Resour Res, 1972, 8: 1272-1283

20 田富强, 胡和平. 基于常微分方程求解器的 richards 方程数值模型. 清华大学学报(自然科学版), 2007, 47: 4

21 田富强, 高龙, 胡和平. 基于 cvode 求解器的饱和-非饱和土壤水分运动数值模型. 中国科学: 技术科学, 2012, 42: 311-324

22 Tromp-Van Meerveld H J, Mcdonnell J J. Threshold relations in subsurface stormflow: 2 the fill and spill hypothesis. Water Resour Res, 2006, 42: W2411

23 刘金涛, 梁忠民. 坡地径流入渗机制对水文模拟的影响分析. 水科学进展, 2009, 20: 467-472

24 Liu D, Tian F, Hu H, et al. The role of run-on for overland flow and the characteristics of runoff generation in the loess plateau. China Hydrol Sci J, 2012, 57: 1107-1117

25 Kazezyılmaz-Alhan C M, Medina Jr M A, Rao P. On numerical modeling of overland flow. Appl Math Comput, 2005, 166: 724-740

26 雷志栋, 杨诗秀, 谢森传. 土壤水动力学. 北京: 清华大学出版社, 1988

27 Sisson J B. Drainage from layered field soils: fixed gradient models. Water Resour Res, 1987, 23: 2071-2075

28 Mccord J T. Application of second-type boundaries in unsaturated flow modeling. Water Resour Res, 1991, 27: 3257-3260

29 van Genuchtech M T. A closed-form equation for predicting the hydraulic conductivity of unsaturated soils. Soil Sci Soc Am J, 1980, 44: 892-898 
30 Pan L, Wierenga P J. A transformed pressure head-based approach to solve richards' equation for variably saturated soils. Water Resour Res, 1995, 31: 925-931

31 Pan L, Warrick A W, Wierenga P J. Finite element methods for modeling water flow in variably saturated porous media: Numerical oscillation and mass-distributed schemes. Water Resour Res, 1996, 32: 1883-1889

32 Hao X, Zhang R, Kravchenko A. A mass-conservative switching method for simulating saturated-unsaturated flow. J Hydrol, 2005, 311: 254-265

33 Sadegh Z K. Multi-scale inverse modeling in biological mass transport processes. Dissertation of Doctoral Degree. Maryland: University of Maryland at College Park, 2006

34 Sadegh Z K. A mass-conservative switching algorithm for modeling fluid flow in variably saturated porous media. J Comput Phys, 2011, 230: 664-679

35 Amestoy P, Duff I, L'Excellent J, et al. A fully asynchronous multifrontal solver using distributed dynamic scheduling. SIAM J Matrix Anal Appl, 2001, 23: 15-41

36 Tromp-Van Meerveld H J, James A L, Mcdonnell J J, et al. A reference data set of hillslope rainfall-runoff response, panola mountain research watershed, united states. Water Resour Res, 2008, 44: W6502

37 Kosugi K, Katsura S, Katsuyama M, et al. Water flow processes in weathered granitic bedrock and their effects on runoff generation in a small headwater catchment. Water Resour Res, 2006, 42: W2414

38 Tromp V M I, Mcdonnell J J. Comment to spatial correlation of soil moisture in small catchments and its relationship to do minant spatial hydrological processes. J Hydrol, 2005, 303: 307-312 